\title{
COMPORTAMENTO DA PRESSÃO ARTERIAL E COMPOSIÇÃO CORPORAL APÓS DOIS TIPOS DE TREINAMENTOS PERIODIZADOS EM PARTICIPANTES COM SÍNDROME METABÓLICA. ENSAIO CLÍNICO CONTROLADO RANDOMIZADO
}

Hygor Ferreira da Silva, Carlos Iván Mesa Castrillón, Maria Paula Ferreira de Figueiredo, Franciele Marquez Vanderlei, Stephanie Nogueira Linares, Leonardo Kesrouani Lemos, Rodolfo Brisola Rodrigues Hidalgo, Alan Marcel Milanez, Natanael Pereira Batista, Carlos Marcelo Pastre, Jayme Netto Junior

Universidade Estadual Paulista - UNESP, Departamento de Fisioterapia, Presidente Prudente, SP. e-mail: hygoratl@gmail.com

\section{RESUMO}

Os treinamentos aeróbio intervalado e resistido são utilizados na prevenção e tratamento da Síndrome Metabólica (SMet), porém há lacunas quanto às diferentes metodologias aplicadas. $O$ objetivo foi analisar e comparar o comportamento da pressão arterial após treinamentos periodizado aeróbio e resistido em adultos com Síndrome Metabólica. Vinte e nove voluntários, sedentários de ambos os sexos, com idade entre 35 e 60 anos com SMet, foram aleatorizados em 3 grupos: treinamento aeróbio intervalado (TAl; $n=9$ ), treinamento resistido (TRC; $n=11$ ) e o grupo controle $(n=9)$. O programa foi constituído por 3 sessões semanais durante 12 semanas. Antes e após o treinamento foi mensurada a pressão arterial em repouso. Houve diminuição da pressão arterial sistólica de $7 \mathrm{mmHg}$ no grupo TAl, sem significância estatística. Não foram observadas alterações na pressão arterial diastólica. Após ambos treinamentos periodizados não foram observados mudanças na pressão arterial em participantes com SMet.

Palavras-chave: Síndrome metabólica, pressão arterial, treinamento de resistência, exercício.

\section{BEHAVIOR OF BLOOD PRESSURE AND BODY COMPOSITION AFTER TWO TYPES OF PERIODIZED TRAINING IN PARTICIPANTS WITH METABOLIC SYNDROME. RANDOMIZED CONTROLLED CLINICAL TRIAL}

\begin{abstract}
Aerobic interval and resistance training are used for prevention and treatment of metabolic syndrome (SMet) however, there are gaps in their methodological applicability. The objective was to analyze and to compare the effects of a model of periodization training, aerobic interval and resistance in blood pressure (BP) in adults with SMet. Twenty nine sedentary volunteers men and women, aged 35 to 60 years with SMet were randomized into 3 groups: aerobic interval training (AIT; $n=9$ ), resistance training (TRC; $n=11$ ) and the control group ( $n=9)$. The program consisted of three sessions per week during 12 weeks. Before and after training was measured blood pressure at rest. There was a decrease of $7 \mathrm{mmHg}$ systolic blood pressure in the AIT group without statistical significance. No changes were observed in the diastolic blood pressure. After both periodized training not changes in blood pressure were observed in people with MetS. Keywords: Metabolic syndrome, blood pressure, resistance training, exercise.
\end{abstract}

\section{INTRODUÇÃO}

A Síndrome Metabólica é um conjunto de fatores de risco para doenças cardiovasculares e diabetes mellitus tipo 2. Caracteriza-se por aumento da pressão arterial (sistólica $\geq 130 \mathrm{mmHg}$ ou diastólica $\geq 85 \mathrm{mmHg}$ ), obesidade central ( $>90 \mathrm{~cm}$ homens e $>80 \mathrm{~cm}$ mulheres), triglicérides ( $\geq 150 \mathrm{mg} / \mathrm{dL}$ ), HDL-colesterol $(<40 \mathrm{mg} / \mathrm{dL}$ homens e $<50 \mathrm{mg} / \mathrm{dL}$ mulheres) e glicemia de jejum $(\geq 100 \mathrm{mg} / \mathrm{dL})^{1}$. Este conjunto de fatores estão associados à mortalidade prematura, aumentando o risco de morte em até 1,5 vezes ${ }^{2}$.

A prevalência da Síndrome Metabólica vem aumentando mundialmente em conjunto à obesidade e diabetes, sendo frequente na população adulta brasileira abrangendo entre 25 a $30 \%{ }^{3}$, isso pode estar relacionado com hábitos de vida e alimentares ${ }^{4}$. 
A atividade física mostrasse eficiente para tratamento dos fatores da Síndrome Metabólica ${ }^{5}$. O exercício aeróbio e resistido demonstram efeitos positivos para glicemia em jejum e triglicérides, circunferência abdominal e pressão arterial $^{6-7}$. Contudo são limitados os estudos que analisam os fatores da síndrome em relação com o treinamento aeróbio intervalado. Por outro lado limitados estudos têm relatado os efeitos de um programa de treinamento aeróbio intervalado e resistido em um modelo periodizado, que facilite a adaptação ao exercício. Em síntese, como indicado anteriormente, o treinamento físico é recomendado na prevenção e tratamento da Síndrome Metabólica, porém há escassez de estudos comparando qual tipo de treinamento (aeróbio intervalado ou resistido) é mais efetivo na redução da pressão arterial ${ }^{5}$. A partir do exposto, o estudo tem o objetivo de analisar 0 comportamento da pressão arterial e a composição corporal após treinamento intervalado e resistido em adultos com Síndrome Metabólica.

\section{MÉTODOS}

\section{População do estudo}

Quarenta e nove (49) voluntários foram avaliados para elegibilidade, destes, 4 participantes não atenderam os critérios de inclusão, incluindo na randomização nos grupos TAI (Treinamento aeróbio intervalado) $n=15, T R C$ (Treinamento resistido convencional) $n=15$ e o grupo controle $n=15$. Este projeto de pesquisa foi submetido ao Comitê de Ética em Pesquisa da Universidade Estadual Paulista "Júlio de Mesquita Filho", Campus de Presidente Prudente e foi aprovado sob o número de CAAE: 31687114.8.0000.5402. Os participantes assinaram o termo de consentimento livre e esclarecido.

\section{Critérios de inclusão}

I. Ter idade entre 35 e 60 anos;

II. Ser diagnosticado com três fatores dos cinco que caracterizam a Síndrome Metabólica ${ }^{1}$;

III. Não fazer atividade física regularmente 3 meses antes da entrevista;

IV. Não apresentar uso frequente de álcool e entorpecentes.

\section{Critérios de exclusão}

I. Realizar menos do $80 \%$ do programa de treinamento

II. lesões osteomusculares que impeçam o treinamento

\section{Delineamentos do estudo}

Os protocolos de treinamento foram executadas com três sessões semanais, totalizando 36 sessões de treinamento. Cada sessão teve a duração entre 30 a 75 minutos, realizadas com intervalo mínimo de 24 horas e máximo de 72 horas entre elas. Todos os voluntários foram orientados a manterem a sua dieta e atividades diárias normalmente. $\mathrm{O}$ grupo controle não realizou nenhuma intervenção. O grupo TAl foi submetido ao treinamento periodizado em esteira ergométrica e o grupo TRC foi submetido ao treinamento resistido periodizado; ambos considerando o princípio do treinamento da inter-relação volumeintensidade.

$$
\text { O TAl foi executado de forma }
$$
individualizada segundo a intensidade, e para sua realização foi pautada o percentual de Frequência cardíaca de reserva (FCR) de acordo com o consenso da Sociedade Europeia de Cardiologia de $2013^{9}$. Sendo dividida em 3 fases: 20-39\% (leve), $40-59 \%$ (moderada) e 60-90\% (alta) da FCR. A pausa entre as séries (recuperação) foi ativa, com tempo variando entre 1 e 4 minutos, até que o participante chegasse aos valores $\leq 19 \%, \leq 30 \%$ à $\leq 50 \%$ da FCR, de acordo com as fases de intensidade leve, moderada ou alta respectivamente. Em cada fase de treinamento houve uma semana recuperativa, caracterizada por repouso total (Tabela 1 ).

Para o cálculo da intensidade de treinamento pela FCR foi utilizada a fórmula: FCR $=(F C m a ́ x-F C r e p) x \%$ treinamento $+F C r e p$, onde $(\mathrm{FCmax}=$ frequência cardíaca máxima; $\mathrm{FCrep}=$ frequência cardíaca de repouso; \% treinamento = percentual de treinamento). Para o cálculo da FCmáx foi utilizada a fórmula de Karvonen ${ }^{9}$, e para a mensuração da FCrep foi utilizado cardiofrequencímetro da marca Polar ${ }^{\circledR}$, em repouso por 5 minutos na posição deitada. Para os pacientes que fazem 0 uso de betabloqueadores foi feita a correção para a Frequência Cardíaca ${ }^{9}$. 
Tabela 1. Dinâmica de cargas (número de séries e repetições, tempo de esforço, tempo de recuperação, tempo total e intensidade de esforço) do treinamento aeróbio intervalado.

\begin{tabular}{|c|c|c|c|c|}
\hline Fases & Semanas & Sessões & $\begin{array}{l}\text { Série } x \text { tempo de } \\
\text { esforço em } \\
\text { minutos }\end{array}$ & $\begin{array}{l}\text { Intensidade } \\
\% \text { da FCR }\end{array}$ \\
\hline \multirow{6}{*}{ Adaptativa } & \multirow{3}{*}{$\begin{array}{c}1-2 \\
3\end{array}$} & $1^{a}$ e $2 \underline{a}$ & $5 \times 4$ & \multirow{3}{*}{$\begin{array}{c}\text { Treino: } \\
\text { 20-39\% da FCR }\end{array}$} \\
\hline & & $3 \underline{a}$ e 4a & $6 \times 4$ & \\
\hline & & 5a e 6ạ & $7 \times 4$ & \\
\hline & \multirow{2}{*}{4} & $7 \underline{a}, 8 \underline{a}$ e $9 \underline{a}$ & $8 \times 4$ & \multirow{2}{*}{$\begin{array}{l}\text { Recuperação: } \\
\leq 19 \% \text { da FCR }\end{array}$} \\
\hline & & $10^{a}, 11^{a}$ e $12^{a}$ & $9 \times 4$ & \\
\hline & \multicolumn{3}{|c|}{ Recuperativa } & \\
\hline \multirow{5}{*}{ Intermediária } & \multirow{4}{*}{$\begin{array}{c}6-7 \\
8\end{array}$} & $13^{a}$ e $14 \underline{a}$ & $4 \times 2,5$ & \multirow{5}{*}{$\begin{array}{c}\text { Treino: } \\
\text { 40-59\% da FCR } \\
\text { Recuperação } \\
\leq 30 \% \text { da FCR }\end{array}$} \\
\hline & & $15 \underline{a}$ e $16 \underline{a}$ & $5 \times 2,5$ & \\
\hline & & $17^{a}$ e $18^{a}$ & $6 \times 2,5$ & \\
\hline & & $19 \underline{a}, 20^{a}$ e $21^{a}$ & $7 \times 2,5$ & \\
\hline & 9 & \multicolumn{2}{|c|}{ Recuperativa } & \\
\hline \multirow{5}{*}{ Avançada } & \multirow{5}{*}{$\begin{array}{c}10-11 \\
12\end{array}$} & $22 \underline{a}$ e $23 a$ & $5 \times 1,5$ & \multirow{3}{*}{$\begin{array}{l}\text { Treino: } \\
\text { 60-90\% da FCR }\end{array}$} \\
\hline & & $24 \underline{a}$ e $25 \underline{a}$ & $6 \times 1,5$ & \\
\hline & & $26 \underline{a}$ e $27 \underline{a}$ & $7 \times 1,5$ & \\
\hline & & $28 \underline{a}$ e 29a & $8 \times 1,5$ & Recuperação \\
\hline & & 30 ạ & $9 \times 1,5$ & $\leq 50 \%$ da FCR \\
\hline
\end{tabular}

FCR: Frequência cardíaca de reserva.

O grupo TRC realizou 3 exercícios para membros inferiores, (legpress, cadeira extensora e cadeira flexora) e cinco exercícios para membros superiores (peitoral, costas, ombro, bíceps e tríceps). 0 treinamento foi executado de forma progressiva e individualizada, e para sua realização a intensidade foi pautada em percentual de 1 repetição máxima (1RM) $)^{9}$. A pausa entre as séries variou entre 40 e 60 segundos (Tabela 2).

Tabela 2. Dinâmica de cargas (número de séries e repetições, tempo de esforço, tempo de recuperação, tempo total e intensidade de esforço) do treinamento resistido convencional.

\begin{tabular}{ccc}
\hline Semanas & Séries x Repetições & \% de 1RM \\
\hline 1 & $2 \times 12$ & $30-40$ \\
\cline { 2 - 3 } 2 & $2 \times 16$ & $30-40$ \\
\cline { 2 - 3 } 3 & $2 \times 20$ & $30-40$ \\
\hline 4 & \multicolumn{3}{c}{ Recuperativa } \\
\hline 5 & $1 \times 16 / 12 / 9$ & $40 / 50 / 60$ \\
\hline 6 & $1 \times 12 / 9 / 6$ & $50 / 60 / 70$ \\
7 & $1 \times 10 / 8 / 6$ & $60 / 70 / 80$ \\
\hline 8 & $1 \times 8 / 6 / 4$ & $70 / 80 / 90$ \\
\hline 9 & & Recuperativa \\
\hline 10 & $1 \times 6 / 4 / 2 / 4 / 6$ & $80 / 90 / 100 / 90 / 80$ \\
11 & $1 \times 6 / 4 / 2 / 2 / 4 / 6$ & $80 / 90 / 100 / 100 / 90 / 80$ \\
12 & $1 \times 6 / 4 / 2 / 2 / 2 / 2 / 4 / 6$ & $80 / 90 / 100 / 100 / 100 / 90 / 80$ \\
\cline { 2 - 3 } & &
\end{tabular}

$1 \mathrm{RM}=$ Uma repetição máxima. 


\section{Variáveis do estudo e coleta de dados Pressão Arterial}

A pressão arterial foi mensurada em repouso de cinco minutos na posição sentada, por meio de esfigmomanômetro aneroide da marca Tycos/WelchAllyn, modelo DS-44 e estetoscópio da marca Litmann, modelo Classic II. Foi orientado aos participantes não fazerem uso de estimulantes como álcool, bebidas que contém cafeína, refrigerante, chocolate, dentre outras, por 24 horas antecedentes à avaliação.

\section{Composição corporal}

As variáveis antropométricas de peso e índice de massa corpórea e composição corporal foram coletadas, por meio de análise da impedância bioelétrica, por balança da marca TANITA, modelo BC - 418, Segmental Body Composition Analyzer, Iron Man/Inner Scaner de 8 canais. Os indivíduos foram orientados a realizar um jejum de 12 horas, tirar todos adornos e vestirem o mínimo de roupa possível (short para homem e top e short para mulheres).

\section{Análise de dados}

Para análise dos dados foi utilizado o poder de teste de $80 \%$. A comparação entre grupos foi feita pelo teste One-way ANOVA com pós-teste de Tukey, e para a comparação entre momentos foi utilizado o teste $t$ de Student pareado. Todos os dados foram analisados com 5\% de significância.

\section{RESULTADOS}

Houve desistências e/ou lesões musculares, sendo assim, ao final, os grupos foram TAI $n=9, \operatorname{TRC} n=11$ e controle $n=9$.

$\mathrm{Na}$ Tabela 3 estão expostos os dados das medidas antropométricas e idade. Não foi observada diferenças entre os grupos em relação a idade e estatura. Houve diminuição $(p<0,05)$ em relação a massa corpórea do grupo TAl, em comparação ao grupo controle nos momentos pré e pós treinamento. Verificou-se diminuição $(p<0,05)$ na variável IMC do grupo TAI e TRC em relação ao grupo controle no momento pré do treinamento.

$\mathrm{Na}$ Tabela 4 foram expostos dados segundo os momentos pré e pós do treinamento em relação a Pressão Arterial (PA) dos voluntários, sem diferença estatística.

Tabela 3. Dados antropométricos.

\begin{tabular}{|c|c|c|c|}
\hline & TAI & TRC & CONTROLE \\
\hline Idade(anos) & $54,444 \pm 3,206$ & $51,272 \pm 5,901$ & $49,888 \pm 5,883$ \\
\hline Estatura $(\mathrm{cm})$ & $171 \pm 9,110$ & $166,82 \pm 11,797$ & $167,222 \pm 11,872$ \\
\hline \multicolumn{4}{|c|}{ Massa Corpórea $(\mathrm{kg})$} \\
\hline pré & $97,311 \pm 17,008^{*}$ & $90,491 \pm 12,902$ & $77,700 \pm 13,884$ \\
\hline pós & $96,555 \pm 17,463^{*}$ & $90,982 \pm 12,966$ & $78,589 \pm 14,956$ \\
\hline$\Delta$ & $-0,755 \pm 1,600$ & $0,490 \pm 1,219$ & $0,888 \pm 1,562$ \\
\hline \multicolumn{4}{|l|}{ IMC } \\
\hline pré & $33,278 \pm 4,970 *$ & $32,566 \pm 4,130^{*}$ & $27,733 \pm 3,722$ \\
\hline pós & $33,026 \pm 5,203$ & $32,727 \pm 4,001$ & $28,012 \pm 3,850$ \\
\hline$\Delta$ & $-0,522 \pm 0,4774$ & $0,1609 \pm 0,4715$ & $0,2789 \pm 0,4805$ \\
\hline \multicolumn{4}{|c|}{$\begin{array}{l}\text { Valores apresentados em média. TAl: Treinamento aeróbio intervalado; TRC: Treinamento } \\
\text { resistido convencional; CONTROLE: grupo controle: } \Delta \text { : média da diferença (final-inicial); } \\
\text { IMC: Índice de massa corpórea; * Diferença estísticamente significante em relação ao } \\
\text { grupo controle }(\mathrm{p} \leq 0,05) \text {. }\end{array}$} \\
\hline
\end{tabular}


Tabela 4. Dados da pressão arterial.

\begin{tabular}{lcccc}
\hline & \multicolumn{1}{c}{ TAI } & TRC & CONTROLE \\
\hline PAS & & & \\
& pré & $130,00 \pm 16,583$ & $116,66 \pm 11,738$ & $117,40 \pm 15,706$ \\
& pós & $122,22 \pm 10,639$ & $116,97 \pm 14,565$ & $118,51 \pm 20,819$ \\
& $\Delta$ & $-7,778 \pm 11,211$ & $0,3045 \pm 17,66$ & $1,114 \pm 25,220$ \\
PAD & & & \\
& pré & $84,444 \pm 14,240$ & $75,756 \pm 10,338$ & $74,069 \pm 11,402$ \\
& pós & $85,000 \pm 9,354$ & $76,059 \pm 8,796$ & $76,280 \pm 15,678$ \\
& $\Delta$ & $0,556 \pm 11,304$ & $0,3027 \pm 1,378$ & $2,211 \pm 16,508$ \\
\hline Valores de PAS e PAD expressos em mmHg; PAS: pressão arterial sistólica; PAD: pres- \\
\multicolumn{2}{l}{ são arterial diastólica; $\Delta$ : média da diferença (final- inicial). } \\
\hline
\end{tabular}

\section{DISCUSSÃO}

A partir dos resultados foi possível constatar que houve diminuição média de 7,77 $\mathrm{mmHg}$ na pressão sistólica no grupo TAl após o treinamento, mesmo que esses resultados não sejam significantes estatisticamente, observa-se influência do TAI no comportamento da pressão arterial sistólica em adultos sedentários com Smet, constatando melhora clínica. No grupo TRC não houve alteração na pressão arterial entre os momentos nem entre grupos.

Em relação aos efeitos do TAl na pressão arterial, os achados de Stensvold et al. ${ }^{10}$ confirmaram os mesmos efeitos, com melhora de aproximadamente $6 \mathrm{mmHg}$ na pressão arterial sistólica, sem significância estatística. Já no estudo de Sari-Sarraf et al. ${ }^{6}$ nota-se melhora significativa da pressão arterial sistólica no grupo TAl, em que foi observado aumento da produção de óxido nítrico (vaso dilatador), porém essa indicação só foi possível entre as 8ạ e 16ạ semanas, a intensidade do exercício foi iniciada a uma carga de $60 \%$ da RCF e depois foi aumentada para $75 \%$ mantendo-se até o final. A não obtenção do resultado esperado pode estar relacionado à intensidade do exercício, pois em nosso estudo iniciamos o treinamento em intensidade baixa alcançando alta intensidade apenas no final da intervenção.

Já para os resultados observados no grupo TRC, contrariamente aos nossos achados, o estudo da Yavari et al. ${ }^{11}$ obteve diferença significativa na PAS e PAD. Esse programa de treinamento iniciou na 1 a semana com $60 \%$ de $1 \mathrm{RM}$ e depois progrediu para $75-80 \%$ de $1 \mathrm{RM}$, tendo duração de 52 semanas. Não termos a diferença significativa da PA no grupo TRC pode ter se dado pelo tempo de intervenção que foi muito curto comparado ao utilizado no estudo de Yavari et al. ${ }^{11}$.

Por outro lado, os resultados observados do IMC revelam aumento no grupo controle e redução do grupo TAl, não significante estatisticamente. Porém, nos estudos de SariSarraf et $a l^{6}{ }^{6}$ e Stensvold et $a l^{10}$ é descrita a melhora do IMC dos grupos TAI e TRC que pode ter se dado pela alta intensidade dos treinamentos, durante o tempo de intervenção. Mostrando que a alta intensidade foi determinante para a diminuição do IMC. Contudo, o treinamento que aplicamos, tinham uma intensidade crescente, só alcançando uma alta intensidade nas últimas três semanas, o que pode explicar a não redução significativa do IMC.

\section{CONCLUSÃO}

Conclui-se que ambos os treinamentos periodizados não diminuíram significativamente a pressão arterial, ainda que o grupo TAl teve uma maior tendência na redução da pressão arterial comparado ao grupo TRC em adultos sedentários com síndrome metabólica.

\section{AGRADECIMENTOS}

À Fundação de Amparo à Pesquisa do Estado de São Paulo (FAPESP) pelo suporte financeiro

\section{CONFLITOS DE INTERESSE}

Os autores declaram não haver qualquer 
potencial de conflito de interesse que possa interferir na imparcialidade deste trabalho científico.

\section{REFERÊNCIAS}

1. Alberti KGMM, Robert $H$, Scott $M$, Paul Z, James I, Karen A. et al. Harmonizing the Metabolic Syndrome A Joint Interim Statement of the International Diabetes Federation Task Force on Epidemiology and Prevention; National Heart, Lung, and Blood Institute; American Heart Association; World Heart Federation; International Atherosclerosis Society; and International Association for the Study of Obesity. Circulation. 2009;120(16):1640-5. DOI: http://dx.doi.org/10.1161/CIRCULATIONAHA.109. 192644

2. Gami AS, Witt BJ, Howard DE, Erwin PJ, Gami LA, Somers VK et al. Metabolic syndrome and risk of incident cardiovascular events and death: a systematic review and meta-analysis of longitudinal studies. J Am Coll Cardiol. 2007;49(4):403-14. DOI: https://doi.org/10.1016/i.jacc.2006.09.032

3. Salas-Romero $R$, Sánches Muños $V$, Franco sánchez JG, Villar Morales A, Pegueros Péres A et al. Effectiveness of two aerobic exercise programs in the treatment of metabolic syndrome: a preliminary study. Gaceta Médica de Mexico. 2014;150(6):490-8.

4. Jaramillo L, A. Sánchez R, Diaz Z, Cobos N, Bryce A, Z. Parra-Carrillo J et al. Consenso latino americano de hipertensão em pacientes com diabetes tipo 2 e síndrome metabólica. Arq Bras Endocrinol Metab. 2014;58(3):205-25. DOI: https://dx.doi.org/10.1590/0004-2730000003019 5. Ciolac EG, Guimarães GV. Exercício físico e síndrome metabólica. Rev Bras Med Esporte. 2004;10(4):319-24.

DOI: https://dx.doi.org/10.1590/S1517-

\section{9}

6. Sari-Sarraf V, Aliasgarzadeh A, Naderali M-M, Esmaelli $\mathrm{H}$, Naderali EK. A combined continuos and interval aerobic training improves metabolic syndrome risk factors in men. Internacional journal of general medicine. 2015;2015(8):20310. DOI: https://doi.org/10.2147/IJGM.S81938

7. Williams MA, Haskell WL, Ades PA, Amsterdam EA, Bittner $V$, Franklin BA et al. Resistance exercise in individuals with and without cardiovascular disease: 2007 update: a scientific statement from the American Heart Association Council on Clinical Cardiology and Council on Nutrition, Physical Activity, and Metabolism.
Circulation 2007;116(5):572-84 DOI: https://doi.org/10.1161/CIRCULATIONAHA.107.1 85214

8. Arruda PD, asumpção C, Urtado C, Oliveira Dorta L, Rosa N, zabaglia $\mathrm{R}$ et al. Relação entre treinamentos de força e redução de peso corporal. Rev Bras Prescr Fisiol Exerc. 201;4(24): 605-9.

9. Earnest $C P$, Johannsen NM, Swift DL, Gillison FB, Mikus CR, Lucian A, Framer K, Lavie CJ, Church TS. Aerobic and strength training in concomitant metabolic syndrom and type 2 diabetes. Med Sci Sports Exerc. 2014;46(7):1293-301. DOI: http://dx.doi.org.10.1249/MSS.00000000000002 42

10. Stenvold D, Tjonna AE, Skavg AE, Aspenes $S$, Stolen T, Wisloff U, Slordahl SA. Strength training versus aerobic interval training to modify risk factors of metabolic syndrome. J Appl Physiol. 2010;108(4):804-10.

DOI. https://doi.org/10.1152/iapplphysiol.00996.2009 11. Yavari A, Najafipoor F, Aliasghrzadeh A, Niafar $M$, Mobasseri M. Effect of aerobic exercise, resistance training or combined training on glycalmic control and cardio-vascular risk factors in patients with type 2 diabetes. Biol Sport. 2012;29(2):135-43. DOI: http://dx.doi.org/10.5604/20831862.990466

Recebido para publicação em 30/08/2016

Revisado em 31/08/2016

Aceito em 02/09/2016 